\title{
ANALYSIS OF AGRICULTURAL SCENES BASED ON SAR INTERFEROMETRY
}

\author{
G. Nico (1), L. Mascolo (2), A. Pellegrinelli (3), D. Giretti (3), F.M. Soccodato (4), J. Catalão (5) \\ (1)CNR-IAC, Bari, Italy, Email: g.nico@ba.iac.cnr.it \\ (2) DIAN s.r.l., Matera, Italy \\ (3) Università di Ferrara, Dip. di Ingegneria, Italy \\ (4) IAT s.r.l, Roma, Italy \\ (5) Instituto D. Luiz (IDL), FCUL, University of Lisbon, Portugal
}

\begin{abstract}
The aim of this work is to study the temporal behavior of interferometric coherence of natural scenes and use it to discriminate different classes of targets. The scattering properties of targets within a SAR resolution cell depend on their spatial distribution and dielectric constant. We focus on agriculture scenes. In case of bare soils, the radar cross section depends on surface roughness and soil moisture. Both quantities are strongly related to agriculture practices. The interferometric coherence can be modelled as the factorization of correlation terms due to spatial and temporal baselines, terrain roughness, soil moisture and residual noise.

We use multivariate analysis methodologies to discriminate scattering classes exhibiting different temporal behaviors of the interferometric coherence. For each class, the temporal evolution of the interferometric phase and radar cross-section are studied.
\end{abstract}

\section{DATASET AND METHODOLOGY}

We analyze a time series of 30 Cosmo-Sky-Med SAR images $\left(9.6 \mathrm{GHz}, 32^{\circ}\right.$ incidence angle, stripmap, $\mathrm{HH}$ polarized), acquired over the area of Ferrara, Italy, between June 2012 and April 2014. This area, characterized by agriculture fields, is almost flat so reducing the impact of layover and foreshortening effects in SAR images. It is assumed that the processed time series of interferometric SAR images are not affected by terrain displacement effects related to geological phenomena.

We report the results obtained on two different patches captured from the Ferrara scene. Interferograms have been obtained by performing two different analyses. For the first scene (Ferrara1, from now on), images were processed with the Small Global Spatial Baseline (SGSB) approach. The interferogram for the $i$-th image $I_{i}$ in the series was defined as follows:

$$
\phi_{i}=I_{i} \cdot I_{M}^{*}
$$

where $I_{M}^{*}$ is complex conjugate of the (multilooked) master image in the series, chosen in order to minimize the sum of perpendicular baselines with respect to each image in the series. For the second scene (Ferrara2), we performed a Short Global Temporal Baseline (SGTB) analysis, evaluating the interferogram for the i-th image $I_{i}$ in the series as

$$
\phi_{i}=I_{i} \cdot I_{i-1}^{*}
$$

where $I_{i-1}^{*}$ is the previous image in the series. In other words, SGTB analysis consists of interferometrically processing the SAR images by selecting as master and slave images two subsequent images of the time series. In this way we selected interferometric couples with the shortest temporal baseline. For each interferometric couple $I_{i}, I_{j}$, the corresponding map of coherence was evaluated.

In order to study the behavior of the different classes of scatterers in the image, the time series of coherence maps were analyzed by first performing a Principal Component Analysis (PCA) and then finding scatterers with consistent behavior by performing a cluster analysis (k-means). The dimensionality reduction implemented by the PCA allows for nuisance removal and therefore for a more robust cluster analysis. We hypothesize the presence of three classes of scatterers in the scene: a class composed by urbanized areas and two classes of fields.

\section{RESULTS}

\subsection{Small Global Spatial Baseline Analysis}

The results presented in this section have been obtained by processing interferometric and coherence time series relative to Ferrara1 test site. From computations, the image acquired on Apr-2014 resulted the best candidate as master minimizing the global spatial baseline. The amplitude image of the scene (master image only) is shown in Figure 2. Part of cities are visible and fields geometry are barely distinguishable. 

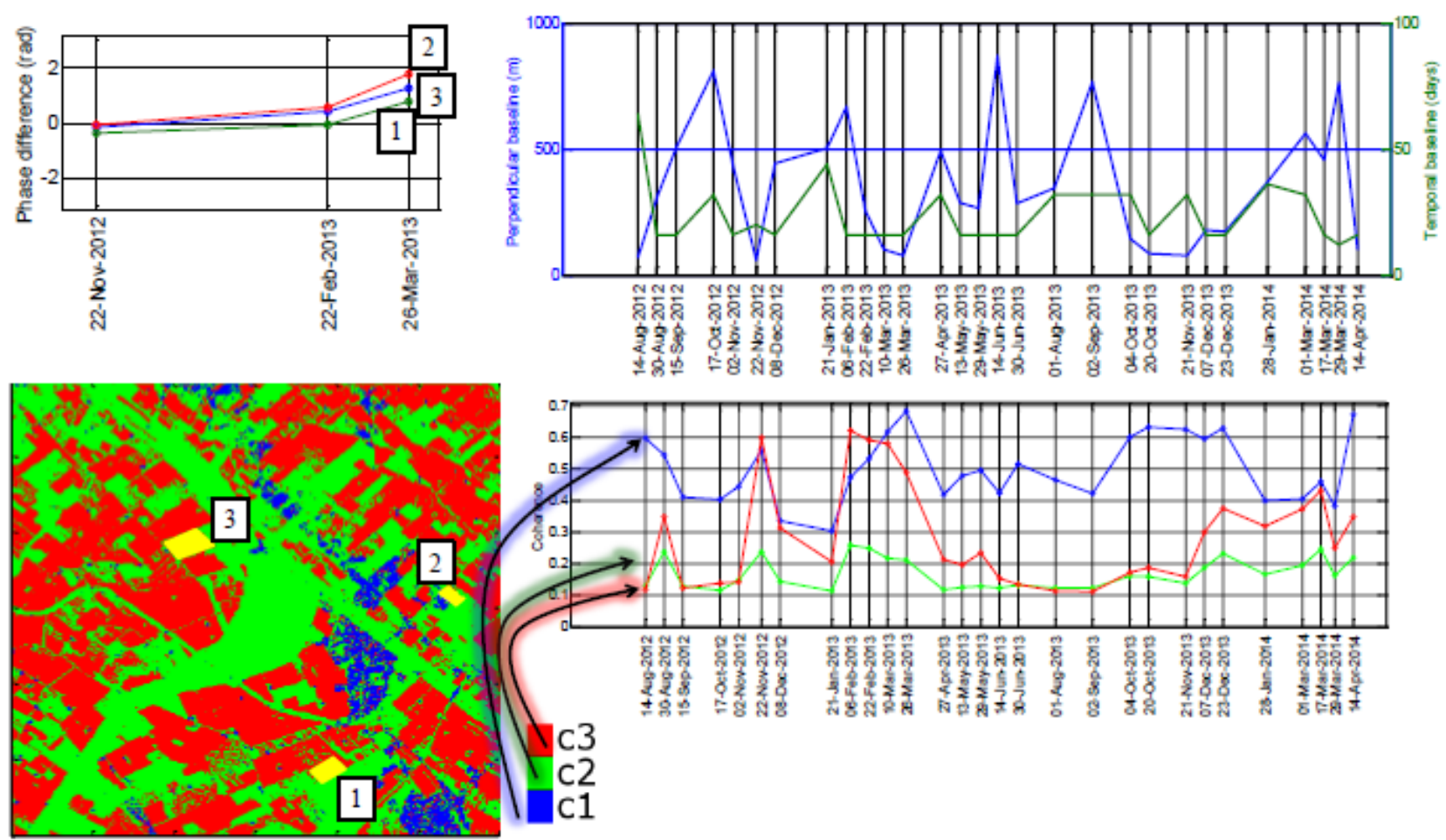

Figure 1: Small Global Spatial Baselines analysis on Ferraral test site. The figure on the bottom left shows the classes identified by a multivariate analysis of interferometric stacks. Figures on the right hand sides represent the spatial and temporal baselines (upper figure) and the mean trends of interferometric coherence associated to the classes identified in the scene (lower figure). Mean interferometric phase values for selected fields are also shown (top left figure).

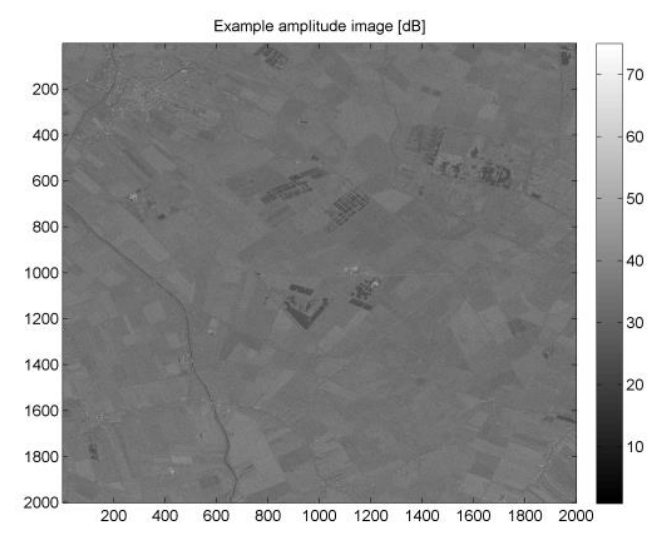

Figure 2. Amplitude image of Ferraral test site.

Spatial PCA was performed on the series of coherence maps relative to the master image. Clustering results have not proved too sensitive to the number of retained components and it can be supposed that most of the useful information for distinguishing the categories of interest is retained by the few first components. We chose to perform cluster analysis on the first four principal components.
Clustering results are shown in Figure 2. The three categories are clearly related to cities and buildings and to two kinds fields. We analyzed separately the mean trend of coherence values for each category. Results are shown in Figure 4, together with the temporal and spatial baselines. Coherence trends associated to cities are clearly strongly related to the perpendicular baseline values: the lower the baseline, the stronger the coherence. Values of interferometric coherence related to fields seem to be correlated more to temporal baseline. In particular, we observe for red marked fields that the average coherence values are higher than the cities ones for small temporal baselines, for 10-Mar-2013 and 26Mar-2013 interferograms. Average values of interferometric phases for 3 selected red marked fields are also reported for 3 dates, characterized by high coherence values. The fields show a similar behavior also in the interferometric phase. 

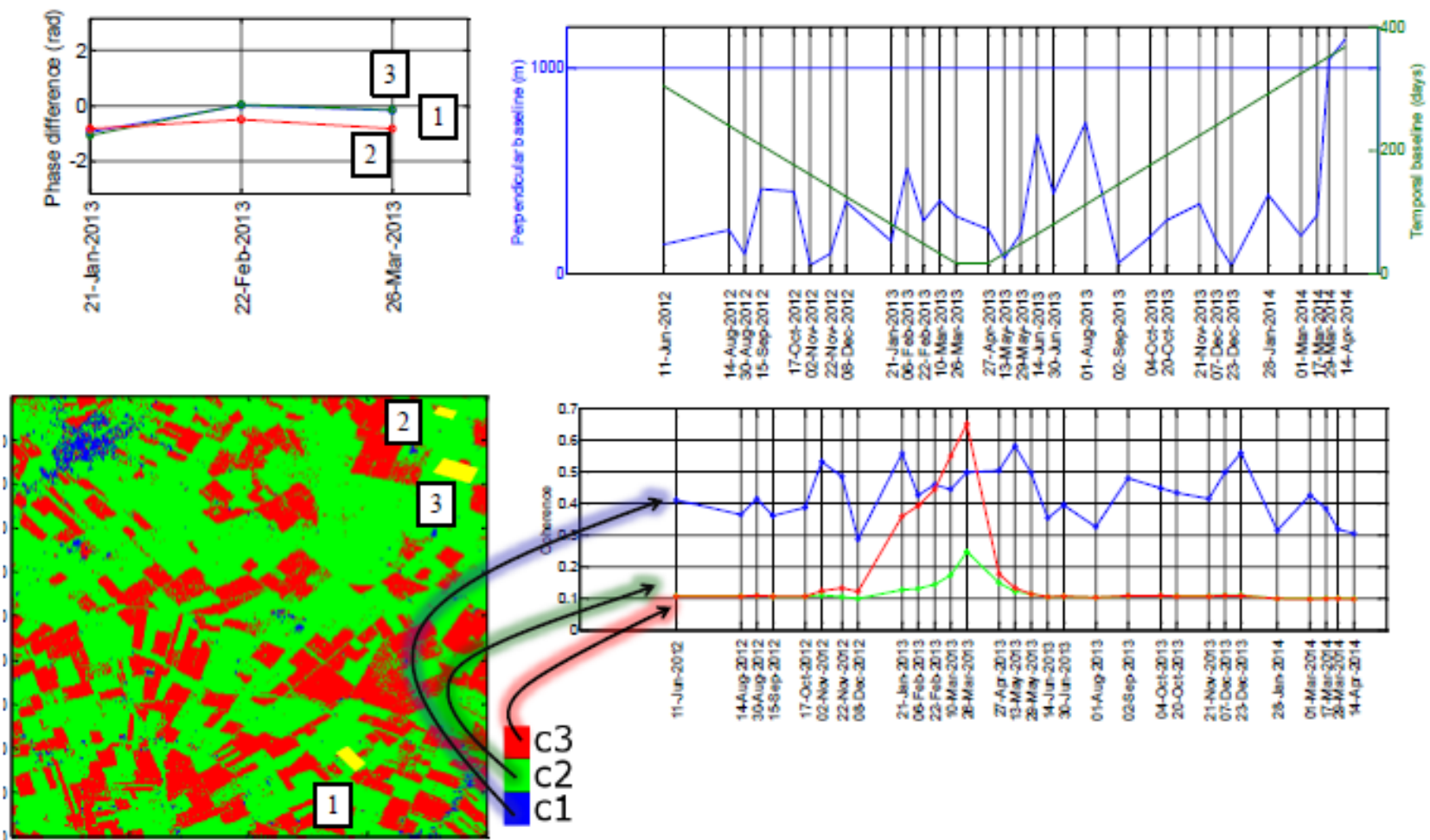

Figure 3: Short Global Temporal Baselines analysis on Ferrara2 test site. The figure on the bottom left shows the classes identified by a multivariate analysis of interferometric stacks. Figures on the right hand sides represent the spatial and temporal baselines (upper figure) and the mean trends of interferometric coherence associated to the classes identified in the scene (lower figure). Mean interferometric phase values for selected fields are also shown (top left figure).

\subsection{Short Global Temporal Baseline Analysis}

Similar processing was performed by using the SGTB analysis. Figure 4 shows the amplitude image of the first acquisition. Also in this case, PCA and cluster analysis were performed (again, 4 components, three clusters) and results are shown in Figure 3. The temporal and spatial

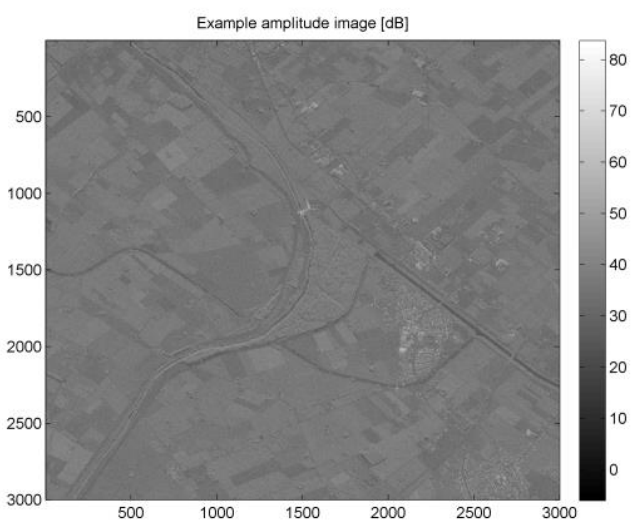

Figure 4. Amplitude image of Ferrara2 test site.

baselines, together with the trends of the three categories captured in the image are plotted in Figure 3. In this case, all three categories show trends of coherence strongly influenced by the perpendicular baseline. Furthermore, strong coherence values, comparable to the city ones, are observed in the period between February-2013 and March-2013. The hole in the trend of coherence observe in 8-Dec-2013 and 21-Jan-2013 is partly due to the perpendicular baseline increase in the same period, but it can be due also to the presence of snow, occurred on 8Dec-2013, that influenced both images. The trend of coherence seems to have an annual period, although it is hard to establish, due to the mixing contribution of the perpendicular baseline.

\section{CONCLUSION}

In this work, a multivariate analysis has been applied to interferometric stacks of SAR image acquired over agricultural fields. We show that it is possible to distinguish between areas characterized by different terrain scattering and/or dielectric properties based on the temporal analysis of maps of interferometric coherence. Results show that two classes of agricultural fields can be identified, possibly related to different agricultural practices such as watering and/or plowing. As a coproduct, the proposed methodology can also identify urbanized areas. Both amplitude and phase variations have been analyzed by the SGTB and SGSB 
methodologies. Future works will focus on the characterization of soil moisture and terrain roughness contributions on interferometric coherence and phase.

\section{REFERENCES}

1. Lopez-Sanchez, J. M., \& Ballester-Berman, J. D. (2009). Potentials of polarimetric SAR interferometry for agriculture monitoring. Radio Science, 44(2).

2. Gabriel, A. K., Goldstein, R. M., \& Zebker, H. A. (1989). Mapping small elevation changes over large areas: differential radar interferometry. Journal of Geophysical Research: Solid Earth (1978-2012), 94(B7), 9183-9191. 